\title{
COMUNIDADES DE APRENDIZAJE PARA LA INCLUSIÓN PLENA EN LOS PROCESOS DE ENSEÑANZA Y APRENDIZAJE DE LOS NIÑOS CON DIVERSIDAD COGNITIVA EN AULAS HETEROGÉNEAS LEARNING COMMUNITIES FOR THE FULL INCLUSION IN TEACHING AND LEARNING PROCESSES OF CHILDREN WITH COGNITIVE DIVERSITY IN HETEROGENEOUS CLASSROOMS
}

\author{
Laura Nataly Galvis Velandia ${ }^{1}$
}

\section{Maritza Carolina Jaimes Márquez ${ }^{2}$}

\section{Raúl Prada Núñez ${ }^{3}$}

UFPS

\section{RESUMEN}

Se acusa la ausencia de un apoyo sistemático al docente de aula para atender y enseñar efectivamente a la heterogeneidad de niños con Necesidades Educativas Exclusivas en un aula integrada junto con los demás niños; lo cual se evidencia por la falta de una $1 \quad$ Magister en Educación, Facultad de Educación, Artes y Humanidades, UFPS. E-mail: lauranatalygv@ufps. edu.co. Orcid: 0000-0003-2444-8398.

2 Magister en Paz, Desarrollo y Resolución de conflictos, Facultad de Educación, Artes y Humanidades, UFPS. E-mail: maritzacarolinajm@ufps.edu.co. Orcid: 0000-00024728-6064.

3 Magister en Ingeniería de análisis de datos, mejora de procesos y toma de decisiones. Facultad de Educación, Artes y Humanidades, UFPS. E-mail: raulprada@ ufps.edu.co. Orcid: 0000-0001-6145-1786 inclusión plena en los procesos de enseñanza $y$ aprendizaje en niños con diversidad cognitiva. Así, se asume el objetivo de diseñar el modelo operativo de las comunidades de aprendizaje, también llamadas comunidades escolares inclusivas, como un recurso de apoyo curricular involucrando, principalmente, a los padres y representantes de los niños con diversidad cognitiva, en la consolidación de las competencias comunicativas y sociales a nivel de los primeros grados de educación elemental en una institución educativa. La metodología parte del proyecto factible desde un enfoque epistemológico pragmatista al seleccionar la investigación mixta. Los resultados alcanzados 
en gran medida confirman las generalidades que son recurrentes en las investigaciones con este tipo de población, aunque sorprendió la evidente desatención a áreas curriculares, prácticamente, inexistentes para los niños en situación. De igual forma, se destaca la absoluta indiferencia e indolencia de los padres y/o representantes de estos niños respecto a su proceso educativo.

Todo lo cual demandó con entusiasmo absoluto de la población consultada la generación de orientaciones teórico-procedimentales como modelo de una comunidad de aprendizaje para superar las debilidades identificadas y fortalecer la responsabilidad social propia de la educación inclusiva.

\section{PALABRAS CLAVE:}

Educación Inclusiva, Comunidades Educativas Inclusivas, Enseñanza para la Diversidad, Discapacidad Cognitiva, Discapacidad Intelectual.

\section{ABSTRACT}

The absence of systematic support for the classroom teacher to effectively attend and teach the heterogeneity of children with Exclusive Educational Needs in an integrated classroom together with the other children, which is evidenced by the lack of full inclusion in the teaching and learning processes of children with cognitive diversity. Thus, the objective of designing the operative model of learning communities, also called inclusive school communities, is assumed as a curricular support resource involving, mainly, parents and representatives of children with cognitive diversity, in the consolidation of communicative and social competences at the level of the first grades of elementary education in an educational institution. The methodology of the feasible project is based on a pragmatist epistemological approach by selecting mixed research. The results achieved largely confirm the generalities that are recurrent in research with this type of population, although the evident neglect of curricular areas, practically non-existent for children in this situation, was surprising. Similarly, the absolute indifference and indolence of the parents and/or representatives of these children with respect to their educational process was also highlighted. All of which demanded with absolute enthusiasm from the consulted population the generation of theoretical-procedural orientations as a model of a learning community to overcome the identified weaknesses and strengthen the social responsibility of inclusive education.

\section{KEYWORDS:}

Inclusive Education, Inclusive Educational Communities, Teaching for Diversity, Cognitive Disability, Intellectual Disability.

\section{INTRODUCCIÓN}

Ante una emergente descentralización y desconcentración administrativa, aún en los países en vía de desarrollo, se corre el riesgo de caer en la anarquía total por falta tanto de la autoridad competente como de una organización eficaz de los ciudadanos. En principio, dentro de toda comunidad, sus miembros se pueden preparar para interactuar cara a cara en procura de satisfacer necesidades, intereses y objetivos comunes, es decir, definidos en forma consensual. De esta realidad no escapan los sistemas educativos donde se ha fracasado al establecer las tradicionales comunidades educativas en gran medida relegadas 0 abandonadas a los padres y representantes (PyR), casi exclusivamente, para financiar las escuelas privadas y subsidiar las públicas.

Así, emerge el estudio de las comunidades de aprendizaje (CoA), que también son llamadas comunidades de práctica (CoP), o comunidades escolares inclusivas (CEI) para procurar cierto equilibrio y equidad en la redistribución de las cargas de información por procesar o volúmenes 
de trabajo a compartir, principalmente, con los docentes de aulas integradas o aulas heterogéneas. Principalmente, los PyR de niños con necesidades educativas exclusivas - NEE (huyendo de la etiqueta de especiales como metáfora de exclusión), deben sensibilizarse y reorientarse con las nociones de desarrollo curricular y estrategias de apoyo directamente demandadas en tiempos de cambio y reformulación de los procesos de enseñanza y aprendizaje (E-A), máxime aun, de las niñas y los niños con serias limitaciones intelectuales o mentales quienes aún no son real y efectivamente incluidos en dichos procesos dentro de aulas integradas desde la educación elemental.

En consecuencia, con esta investigación se desea brindar como aportes algunas luces sobre las comunidades de aprendizaje (CoA), como comunidades de práctica (CoP), y las comunidades escolares inclusivas (CEI), que en lo sucesivo fusionarán sus siglas así, CEl/CoA/ CoP para referirse a una estrategia emergente de desarrollo curricular, cambio e innovación desde el aula de clases donde los expertos recomiendan, hoy día, reubicar a los niños con necesidades educativas exclusivas con el grupo común en escolaridad de los demás niños. Más concretamente, se asume dentro del objeto de estudio a los niños con discapacidad cognitivas (DC) que, se prefiere llamar diversidad cognitiva (DC). Particularmente, interesan aquellos que sufren serias limitaciones pero que no les está vedado ni impedido en términos absolutos un rendimiento escolar satisfactorio dado que sus limitaciones, actualmente, se asocian con los recursos y medios a su alcance o contexto durante su vida que, por cierto, no se restringen tampoco a los docentes ni los centros educativos. Más aún, el foco en las diversidades cognitivas no excluye tampoco a quienes sufren dificultades en el aprendizaje sin saberlo, en la mayoría de los casos, asociadas con limitaciones leves o moderadas en los procesos cognitivos básicos como la atención y la memoria; los cuales, obviamente, se asocian con el bajo rendimiento escolar tan ampliamente reportado en Latinoamérica. Valga reseñar, se prefiere hablar de diversidad cognitiva antes que de discapacidad o discapacitados para superar la connotación irreversible de minusválidos por déficits congénitos y absolutos de esta última noción; la cual no se aplica, hoy día, ni en el caso extremo del retardo mental simbolizado con el Síndrome de Down.

\section{LA DIVERSIDAD COGNITIVA}

La noción de retardo mental ha evolucionado al considerarlo inicialmente como una discapacidad o minusvalía permanente. Aún en los casos más graves, ahora deja de parecer un rasgo absoluto o condición inalterable para entenderse su evolución como el resultado de la interacción del sujeto afectado con un medio o entorno socioeducativo que poco o mucho le ha favorecido. Tal énfasis en el contexto, donde la persona se debe desarrollar, en cuanto se ofrezcan de manera consistente las actividades y los recursos más adecuados a sus circunstancias o desventajas, es congruente con el interés de la autora de la presente investigación.

Pues, se reconocen las deficiencias o los errores del sistema educativo tradicional o el hecho educativo en cuestión cuando se propone el estudio de la viabilidad de implantar una estrategia de apoyo curricular como las CoA, CoP o CEI, cualquiera de las cuales vendría a mejorar las condiciones que actualmente se enfrentan desde los centros de educación inicial los niños con alguna limitación o diversidad cognitiva. Máxime aún, cuando Cabero, Córdoba y Fernández (2008) ratifican que:

Hoy (se entiende) esa diversidad cognitiva como el resultado de las diferencias que el alumnado presenta en cuanto a los procesos cognitivos básicos que subyacen al aprendizaje. Por ello en ningún momento 
(se va) a hablar de retraso mental, ya que la condición de diversidad cognitiva va mucho más allá de esa concepción reduccionista. (Dichos autores) consideran que las diferencias encontradas a la hora de analizar los procesos cognitivos básicos de los alumnos del aula dan como resultado la necesidad de atender a su diversidad cognitiva independientemente de que exista, o no, retraso mental. (p.32-33)

Valga reseñar, cómo puntualizan los autores a continuación, la inquietud compartida en el presente estudio:

Pasando por distintas etapas, se ha pasado al concepto de diversidad, en el que se plantea una concepción de las diferencias individuales, su aceptación y afrontamiento, no como una problemática del sujeto, sino como la necesidad de que el contexto le ofrezca soluciones. (p. 33)

Ciertamente, se evita hablar de retardo mental o discapacidad cognitiva para superar la visión congénita, absolutista y determinista por una concepción basada en las diferencias individuales generadas más por el historial de vida dentro de cierto entorno o contexto familiar y educativo. Más aún, antes que un impedimento insalvable, la diversidad cognitiva enfocada al extremo negativo de las limitaciones en los procesos cognitivos como la atención y la memoria, abarca un conglomerado de personas mucho más amplio; es decir, cualquiera puede sufrir algún tipo de estas limitaciones en sus diferentes grados o niveles.

\section{LA IDEA ESENCIAL DE COMUNIDAD}

Como la define García Aretio (2003), toda comunidad se sustenta en una reconocida forma de relaciones sociales, a ser "considerada como grupo de personas que interaccionan socialmente porque comparten, temporal o permanentemente una serie de intereses comunes" (p.171). Más puntualmente:

En una comunidad se agrupan personas para interactuar socialmente, establecer lazos comunes y compartir ciertos intereses, expectativas, creencias, valores y actividades que establecen los límites e identidad diferenciadora del grupo y todo ello, al menos, durante un tiempo. (p.172)

Dicho autor destaca el carácter social asociado a toda noción de comunidad. Pues, para establecer vínculos y compartir intereses parece esencial su ubicación en un mismo espacio físico como base de su conformación o constitución en un territorio o ámbito organizativo determinado. Aunque no se limita al espacio físico e intereses comunes, tales principios los confirma, citando a Wilbur, García Aretio (2003) cuando agrega que:

También parece aceptado que comunidad proviene del término latino común... hablando de un compartir en común de ideas, objetos, valores, identidades, cualidades y ello dentro de un determinado espacio, también común. La Enciclopedia Universal Larrousse 2003 se refiere a comunidad como un grupo social con intereses, costumbres, opiniones o caracteres comunes y el Diccionario de la Real Academia Española 2002, lo hace como conjunto de personas vinculadas por características o intereses comunes. Sin embargo, parece a algunos que el concepto de comunidad lo definiría mejor el término armonía que el de unidad de conjunto. Así lo señalaba ya Aristoteles en su Etica a Nicómaco... En consecuencia, (cabe) señalar como rasgos y componentes de una comunidad el espacio, la comunicación, la unidad, los intereses, el sentido de pertenencia y la armonía. (p. 171) 
Al respecto, Foster (citado en García Aretio, 2003) destaca que no son elementos esenciales del concepto de comunidad que sus miembros tengan asimilado cierto sentido de pertenencia y compartan un espacio en común. Pues, para estos autores, el elemento comunicacional es esencial dentro del concepto de comunidad. Así advierten que, "puede existir comunicación sin que necesariamente los comunicantes formen parte de la misma comunidad" (p.173). Lo esencial a recordar, según Habermas (citado en García Aretio, 2003), es que, "todas las personas (son) capaces de comunicarse y, en consecuencia, actuar en su entorno, también de forma consensuada". (p.175).

\section{ANÁLISIS DEL CONTEXTO ESCOLAR}

La escuela, como nicho principal de aprendizaje, necesita que, desde la órbita funcional de su operatividad, se generen no sólo conocimientos sino actitudes para enseñar y aprender a ser competente. Así, como ya no se requiere que el maestro asuma su trabajo como el único poseedor del saber sino todo lo contrario, él debe entender que en la sociedad del nuevo milenio su papel radica en ser moderador de conocimientos (López-García y Gutiérrez-Niño, 2018; GarcíaQuintero y Villamizar Suárez, 2017); los cuales son hoy ampliamente difundidos a través de las tecnologías de la información y la comunicación (TIC). Igualmente, la escuela debe garantizar una educación para todos tendiente a la equidad social o igualdad de oportunidades (Stainback, Stainback y Jackson 1999).

Sin embargo, a partir de la entrada en vigencia de la Ley de Inclusión Educativa (Congreso de Colombia, 1996, 1997; Consejería Presidencial para la Política Social, 2002; Ministerio de Educación Nacional, 2006) las instituciones educativas poseen en sus aulas de clase tanto los niños y las niñas regulares como aquellos con marcada diversidad cognitiva, también conocida como discapacidad cognitiva, desde una visión pesimista, así como dificultad para el aprendizaje vista desde el optimismo. Al respecto, no se trata de atacar el problema educativo sólo de niños con retardo mental (ampliamente representado por el Síndrome de Down) sino de otros tantos casos que leve o moderadamente siempre afectan los procesos mentales y el rendimiento escolar, incluso, de niños y niñas aparentemente considerados normales o regulares pero que no suelen alcanzar una actuación escolar satisfactoria.

Sin embargo, los expertos reseñan la escasa difusión sobre la falta de dominio de las metodologías especiales de enseñanza, así como una cultura de mitos o tabúes en torno a este sector de la población. En tal contexto, durante la jornada y cotidianidad escolar, suele acusarse que no se incluyen en sus programaciones y dinámicas escolares a estos niños y estas niñas especiales, aunque estén presentes y a cargo del docente de aula común o integrada. En el mejor de los casos se identifican, entonces, en su enseñanza, procesos que no se corresponden con el tratamiento esperado hacia estos estudiantes; quienes son poseedores de derechos, especialmente, en un sistema educativo que aborda políticas de inclusión como garantía para recibir un aprendizaje e ilustración de acuerdo con las necesidades de cada educando (Cabero, Córdoba y Fernández, 2008; Gamboa Suárez, 2016; Méndez-Omaña y Jaimes-Contreras, 2018).

Para que se puedan implementar estas metodologías con el componente dirigido a los niños y las niñas con diversidad cognitiva, es pertinente que se promuevan procesos de desarrollo curricular, entendido éste como el conjunto de iniciativas dinámicas de acción docente; las cuales tienden a definir estrategias y recursos de apoyo a los procesos de enseñanza y aprendizaje para beneficiar 
a los sectores de la población escolar menos favorecidos (Díaz-Barriga Arceo y Hernández Rojas, 2010). Este ideal de las adaptaciones curriculares, planteado también por los órganos estatales responsables del sistema educativo, no se cumple. En consecuencia, a pesar de ser los principales promotores de la incentivación, promoción y realización de estas propuestas, muchos docentes de aula común no desarrollan las estrategias enunciadas anteriormente para la inclusión plena y relegan a los niños y las niñas con limitación o diversidad cognitiva en sus procesos de enseñanza y aprendizaje.

Precisamente, entre las causas que explican este accionar de los docentes, se identifican ciertos mitos entre los cuales destaca que los maestros poseen una errónea concepción respecto a los niños y las niñas limitados por diversas condiciones cognitivas. Pues, suelen creer que las mismas son discapacidades permanentes e irreversibles $\mathrm{y}$, por tanto, estos niños en ningún momento van a poder interiorizar el conocimiento y llegan a opinar, abiertamente, que siempre van a seguir siendo las mismas personas sin ningún tipo de avance educativo (Cabero, Córdoba y Fernández, 2008)

Además, es esencial agregar que los cuestionables señalamientos contra esta población son esencialmente por culpa de malos maestros. Cuando ellos descuidan y desmeritan la participación de la comunidad en general, se desarrolla de manera latente o expresa este tipo de discriminación. Por su parte, los padres de estos niños con necesidades educativas exclusivas, por su situación DC, cuando están desorientados o desinformados, no participan ni tampoco se comprometen de forma mínima con esta población en las actividades no sólo del aula de clases y la escuela sino aquellas asignadas al grupo familiar, incluso.
El problema que se presenta de acuerdo con lo planteado sobre los niños y las niñas con diversidad cognitiva, es la falta de oportunidades socioeducativas para que ellos reciban orientaciones adecuadas para el aprendizaje, es decir, no se logra su inclusión plena mediante adaptaciones curriculares específicas para los estudiantes con un nivel cognitivo acorde a sus edades y necesidades exclusivas. Por consiguiente, el docente que se limita al desarrollo de las mismas metodologías y rutinas de enseñanza para todos sus estudiantes, hace excluyente de su praxis a la población con diversidad cognitiva, vulnerando el derecho a una educación equitativa o justa.

Esta situación problémica ha generado consecuencias colaterales como la inestabilidad en el entorno familiar, al excluir a los padres y aislar al integrante de la familia que presenta la dificultad en los procesos educativos. Pues, al no encontrar en su hogar ni en la escuela el apoyo y la orientación apropiada para su mejora o superación personal, se genera incertidumbre e incredulidad, desconfianza y hostilidad, principalmente, hacia los maestros y los centros educativos encargados de desarrollar aprendizajes para todos.

Por la necesidad de superar esta situación deficitaria y fraudulenta, surge el problema de estudiar la pertinencia de adoptar, establecer, desarrollar y ejecutar estrategias curriculares para reforzar la atención escolar de la población especial. Pues, se debe mejorar y consolidar la inclusión plena de niños y niñas con alguna limitación o diversidad cognitiva que, a cargo de sólo un docente, están siendo incomunicados, especialmente, de los conocimientos y prácticas de una asignatura como lo es Lengua Castellana. 
De igual forma, estas estrategias deben ser establecidas conforme a la problemática que se está evidenciando, es decir, se deben realizar con profundidad buscando principalmente la integración de esfuerzos para beneficiar esta población infantil, a los efectos de garantizar las competencias comunicativas, en su lengua materna, acordes con la situación geopolítica y social de los estudiantes y las exigencias de atención a la diversidad y equidad.

Así, se hace necesario generar el desarrollo de las CoA, como una herramienta de trabajo pedagógico que le permita a los docentes y padres, promover el uso y la apropiación de estrategias válidas para desarrollar aptitudes en la lectura y la escritura con todos los estudiantes. En efecto, como docentes, se está llamado a utilizar una serie de recursos y métodos para tratar de capturar la atención no sólo del estudiante, por naturaleza siempre dispersa, sino de sus representantes. Sin olvidar que es en la primera infancia y edad preescolar, que transcurre mayormente en el hogar, cuando se fijan y adquieren los conceptos con los que se edifican los pilares de la personalidad y el temperamento.

En su defecto, los docentes innovadores deben estar capacitados para reorganizar de manera intencional el proceso de E-A en diferentes contextos y con diferentes poblaciones de niños y niñas de diversas características. Como cabe esperar, todo docente debe ser capaz de demandar asertivamente los recursos humanos y materiales para redimensionarlos, de manera comprobadamente efectiva, en el proceso E-A bajo su responsabilidad directa. Generando ciertas condiciones para la aplicación y apropiación de conocimientos, el docente de hoy debe orientarse hacia acciones y estrategias que transformen la realidad del proceso E-A dando nuevos sentidos a estructuras, relaciones y contenidos, siempre utilizando para ello el lenguaje en su contexto social como instrumento inexpugnable en toda enseñanza y aprendizaje.
Ciertamente, el lenguaje posee un valor social para el ser humano, en la medida en que le permite establecer y mantener las relaciones interpersonales con sus semejantes; las cuales le posibilitan compartir expectativas, deseos, creencias, valores, conocimientos cuando se construyen espacios conjuntos para su difusión y permanente transformación. Por tal razón, la implementación de una CoA ductiliza dicha tarea, porque a través de ella, se instrumenta sistemáticamente el aprendizaje colaborativo y participativo; con el cual, las competencias comunicacionales fluyen y afloran en función de un aprendizaje para la vida y no para el currículo. Ante esta situación surge las siguientes preguntas: ¿Cuál es el nivel de dominio de las competencias comunicativas y sociales por parte de los niños y niñas con diversidad cognitiva del grado Primero de la Educación Básica Primaria? ¿Cuáles son las condiciones curriculares y administrativas existentes para la enseñanza de la Lengua Castellana a los niños y las niñas con diversidades cognitivas? ¿Cuál será la percepción sobre los niños y las niñas con tales necesidades educativas, así como de sus padres en relación con su inclusión plena en el aula común al desarrollar las estrategias metodológicas para la enseñanza de la Lengua Castellana? ¿Cómo se generaría el desarrollo de CoA como una estrategia curricular innovadora para la inclusión plena en los procesos de enseñanza y aprendizaje de la asignatura de Lengua Castellana de niños y niñas con diversidad cognitiva? 


\section{METODOLOGÍA}

En efecto, en esta investigación se realiza una exploración preliminar mediante la observación diaria en la institución educativa, en dónde se ha evidenciado la necesidad de una inclusión más efectiva de los niños con discapacidad cognitiva en los procesos de enseñanza y aprendizaje junto al grupo común de los primeros grados. En tal sentido, se ha considerado la creación y funcionamiento de una comunidad de aprendizaje, con estrategia de apoyo curricular a dichos procesos. Tal propuesta ha sido fundamentada teóricamente con una investigación documental que se valida con un trabajo de campo para determinar su factibilidad $y$, así, adecuar el diseño de un modelo operativo viable.

A partir de la operacionalización de variables se identifica la utilización de variadas técnicas e instrumentos de recolección de datos coherentes con el enfoque cualitativo de la investigación. Así, se han adaptado los métodos a la naturaleza del objeto de estudio con sus variables y sus correspondientes indicadores; lo cual conduce a asumir epistemológicamente un enfoque pragmatista propio de este método (Hernández Sampieri, Fernández Collado y Baptista Lucio, 2010).

En cuanto al diseño de la investigación, se establece que se deben recoger datos cualitativos en una primera etapa cuyos resultados alimentarán una segunda fase en la cual se precisan, estructuran y analizan datos cuantitativos, por lo que se concluye que se sigue un diseño exploratorio secuencial.

Se identifica como población de interés, la comunidad educativa de docentes, padres o representantes y niños con alguna limitación o diversidad cognitiva que cursaron el grado Primero durante el año 2020 en una institución educativa de naturaleza privada de la ciudad de San José de Cúcuta. De tal manera, cabe distinguir subpoblaciones entre las que destaca aquella conformada por los mencionados padres o representantes como eje central de la comunidad de aprendizaje. Igualmente, están los niños y las niñas con diversidad cognitiva que apenas comienzan la educación primaria integradas en aulas comunes, así como sus docentes y los directivos del plantel seleccionado.

En esta investigación no hay proceso de muestreo dado la población objeto de estudio es pequeña (niños con diversidad cognitiva) por ello tal como lo señala Landeau (2007) no se necesario seleccionar una muestra. En cuanto a los instrumentos para la recolección de datos, se recurre a la utilización de la observación directa del trabajo de aula y la realización de entrevistas semiestructuradas tanto a los expertos en el área como a dos docentes de la institución educativa. Finalmente, se adopta un enfoque descriptivo para el procesamiento de los datos que se recolecten en cada instrumento.

\section{RESULTADOS}

A continuación, se presentan los resultados derivados de este proceso investigativo solo en su fase cualitativa, los cuales han sido organizados de forma lógica y según su intencionalidad.

\section{PROCESO DE VALIDACIÓN}

Los criterios de rigor para la recolección de información cualitativa establecen, entre otros, la cuestión de credibilidad; su búsqueda implicó la presencia de los investigadores durante varias sesiones de observación a los docentes en sus interacciones dentro del aula integrada. Concretamente, tomó varios meses y grupos de estudiantes, el observar las clases impartidas en el aula común de los primeros grados en la institución educativa seleccionada. 
Otro criterio de rigor asumido para el análisis de las notas de observación en el presente informe partió de las lógicas inductivas particulares tendientes a crear unidades de análisis como asuntos puntuales o tópicos que señalan patrones, hechos y fenómenos en correlación estricta con ciertas líneas o párrafos del texto o transcripción de las observaciones.

A continuación, se presentan una serie de cuadros en los cuales, se puede observar que en una columna se describen los pasajes observados puntualizando sus respectivas unidades de análisis. Con base en dichas unidades, la triangulación de fuentes o datos fue posible para construir las categorías conceptuales emergentes directamente de los datos, respecto a las necesidades en cuanto a las competencias comunicativas y sociales identificadas en los niños y las niñas con dificultades cognitivas.

La aplicación de la entrevista semiestructurada se focalizó con un grupo de expertos psicopedagogos quienes fueron abordados con base en un guion de preguntas. Las respuestas y sesiones generadas fueron grabadas en audio y transcritas en texto, lo cual permitió su posterior organización, análisis. Vale la pena reseñar, que no fue necesario ampliar la muestra de expertos por la marcada tendencia a coincidir en sus respuestas.

\section{PROCESAMIENTO DE LA OBSERVACIÓN DIRECTA}

En los siguientes cuadros se muestran los resultados derivados de la fase de observación directa, que realizaron los investigadores durante cuatro sesiones de clase adelantadas por la docente titular del grado Primero. Este proceso se realiza con el objetivo de identificar las necesidades en cuanto a competencias comunicativas y sociales de los niños con DC, en la clase de Lengua Castellana.

En la primera sesión se detectó consistentemente cierto énfasis en el lenguaje mimético y el oral, acompañado de una débil atención al lenguaje escrito, así como a las diferencias individuales entre los dos niños en situación DC, por efecto de su atención dispersa o limitación psicomotora sufrida marcadamente por uno de ellos, desencadenando su escasa interacción social (ver Tabla 1). 
Tabla 1. Notas de campo de la primera observación realizada el 17 de septiembre del 2020

\begin{tabular}{|c|c|}
\hline Unidades de Análisis & Observación \\
\hline $\begin{array}{l}\text { Lenguaje mimético: } \\
\text { Acentuado. }\end{array}$ & $\begin{array}{l}\text { La docente inicia el momento de la oración encomendando la jornada. Revisa la asistencia con los } \\
\text { ausentes. Recuerda lo visto en la clase anterior diciendo que quien quiera hablar pida la palabra. Realiza } \\
\text { las preguntas de acuerdo a lo visto anteriormente (evaluando). }\end{array}$ \\
\hline Lenguaje oral: Acentuado. & $\begin{array}{l}\text { A partir de lo anterior inicia la clase realizando una ambientación de manera general para todo el grupo, } \\
\text { luego aborda la temática que para el día de hoy es el fonema de la consonante W. Dice a los niños: vamos } \\
\text { a observar un video del mono sílabo, y van a repetir los sonidos y las palabras cuando el mono lo pida. }\end{array}$ \\
\hline $\begin{array}{l}\text { Atención dispersa de niño con DC, } \\
\text { carácter retraído, } \\
\text { ensimismado, aislado. }\end{array}$ & $\begin{array}{l}\text { La docente está atenta a captar la atención de todo el grupo. Los dos niños en situación de DC, no logran } \\
\text { fijar la atención por períodos prolongados de tiempo; sin embargo, repiten los sonidos del fonema y las } \\
\text { palabras junto con los otros niños. En tanto que termina el video, uno de los niños con DC se observa } \\
\text { ensimismado, retraído, mientras que el otro empieza a imitar sonidos del video y a producir un ruido } \\
\text { particular uuuuuuuuuuu; frente a dicho comportamiento la profesora reacciona haciéndole un llamado de } \\
\text { atención verbal: No haga eso, silencio, colocando su dedo índice en la boca. Ante el cual el niño responde } \\
\text { positivamente. }\end{array}$ \\
\hline $\begin{array}{l}\text { Lenguaje escrito: Débil. } \\
\text { Diferencias individuales entre } \\
\text { niños con DC. } \\
\text { Uno con limitaciones } \\
\text { psicomotoras. }\end{array}$ & $\begin{array}{l}\text { Luego la profesora pasa por los puestos y dibuja una letra W grande en cada cuaderno y pide a los niños } \\
\text { que la coloreen. Uno de los niños con DC acata la instrucción y su coloreado se observa acorde a un } \\
\text { niño de seis años. Entre tanto el otro niño con DC se queda quieto y solo comienza a colorear cuando la } \\
\text { profesora se dirige a él de forma particular. Su coloreado corresponde a un niño más o menos de cuatro } \\
\text { años, se observa que tiene una limitación motora que le dificulta la forma de agarrar el lápiz y lo hace más } \\
\text { lento. }\end{array}$ \\
\hline Atención dispersa. & $\begin{array}{l}\text { Dando continuidad a la clase, pide que copien lo escrito en el tablero en su cuaderno, a su vez nuevamente } \\
\text { refuerza en forma oral los sonidos de la consonante con las vocales. Los niños con DC repiten lo que la } \\
\text { profesora dice. Pero, el que tiene déficit motor los emite con mayor dificultad y lo hace más tímidamente. } \\
\text { Durante el desarrollo de las actividades, se observa ruido producido por los participantes de la clase y } \\
\text { proveniente del patio de juegos, lo que hace que los estudiantes se distraigan y pierdan la concentración } \\
\text { en lo que hacen, especialmente los niños con DC. Al observar los cuadernos de notas, se aprecia que el } \\
\text { estudiante con déficit motor hizo una transcripción incompleta, con trazos irregulares, omitiendo algunos } \\
\text { grafemas; mientras que el otro niño lo hizo en forma apropiada y al mismo ritmo del resto de la clase. }\end{array}$ \\
\hline $\begin{array}{l}\text { Interacción } \\
\text { docente-estudiante. }\end{array}$ & $\begin{array}{l}\text { Es de anotar que la profesora permanentemente hace llamados de atención verbal para normalizar el } \\
\text { grupo y poder mantener el ritmo de trabajo de los estudiantes. Constantemente, les pide que el trabajo } \\
\text { del cuaderno lo hagan con decoro y organizado. Mientras los estudiantes trabajan en forma individual, } \\
\text { ella camina por entre las filas para verificar que se esté realizando el trabajo y al ver el trabajo de una } \\
\text { estudiante, le orienta qué debe corregir con la finalidad de hacer las cosas bien. Terminada la hora de } \\
\text { clase, la profesora hace un breve receso para iniciar con la otra asignatura. }\end{array}$ \\
\hline
\end{tabular}


En la Tabla 2 se presenta lo sucedido en la segunda clase impartida en el aula común. Se destaca la necesidad de atención en lo concerniente al lenguaje escrito, pues, su enseñanza abarca poco tiempo y esfuerzo durante la clase. Similarmente, no deja de registrarse la limitación respecto al control de la atención como proceso cognitivo básico que es muy inconstante entre los niños en situación de DC. En cuanto a las competencias sociales, se destacan como avances la aceptación, interacción y ayuda que reciben de la docente y de otros niños del curso. Se sigue evidenciado el débil asertividad mostrada por la docente en cuanto al manejo de las necesidades demandadas por los estudiantes en condición de DC.

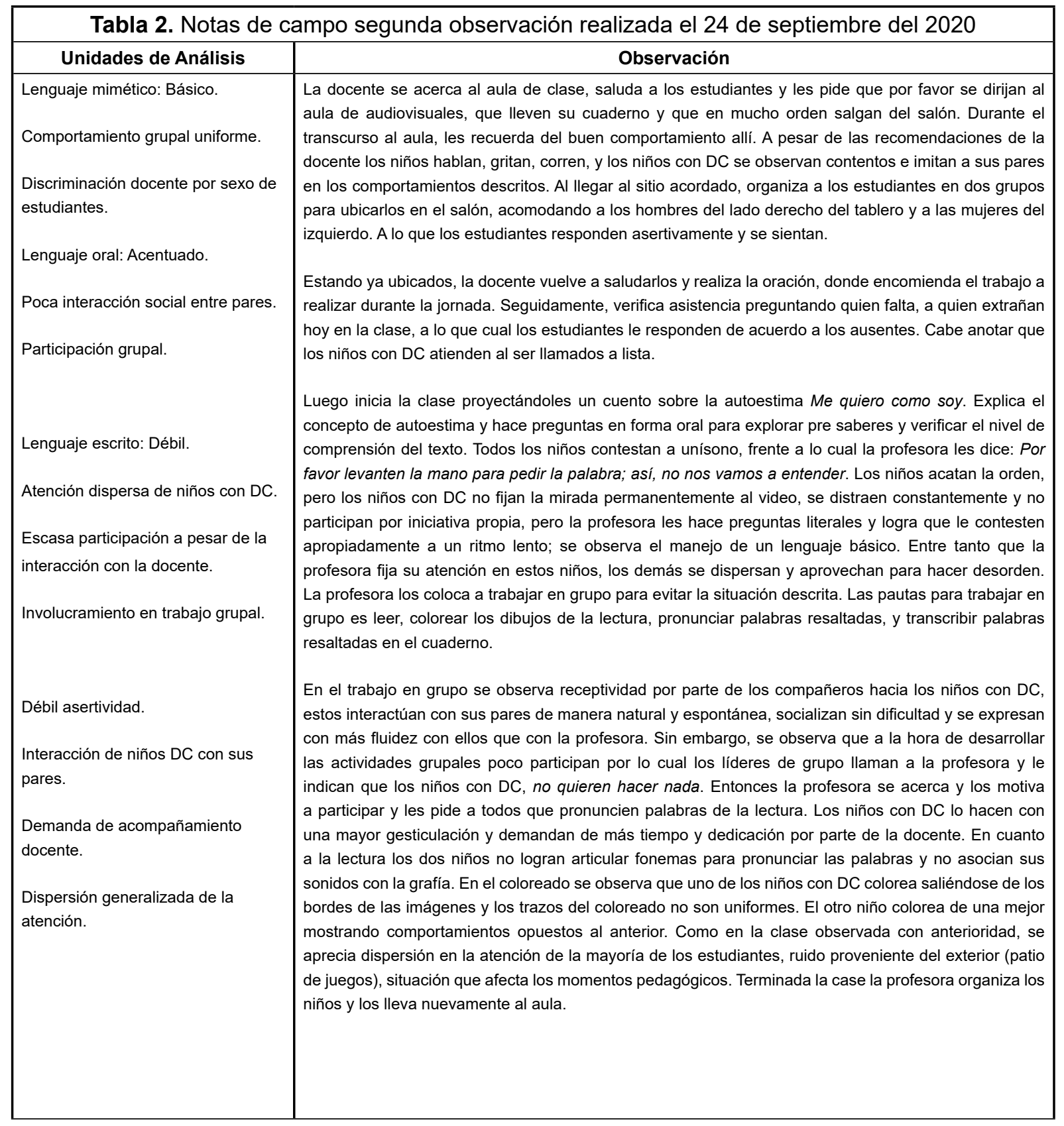


Dos semanas después, se pasa a la tercera clase observada cuyo informe reposa en la Tabla 3, se observa que persiste un deficiente atención u ocupación en la enseñanza de las competencias comunicativas con el lenguaje escrito. También, se reseñó que no se adelantó una evaluación del aprendizaje diferente y adaptada a los niños con DC, es decir, se ignoraron las diferencias individuales y la especial atención que demandan este tipo de estudiantes. Así mismo, se destaca en las observaciones de esta sesión de clases, que dentro de la actividad de clases surgieron reiteradamente incidentes que involucró a un niño con DC y en los que se evidencia que estos niños requieren de mayor atención y mejores recursos de intermediación pedagógica.

\begin{tabular}{|c|c|}
\hline Unidades de Análisis & Observación \\
\hline $\begin{array}{l}\text { Lenguaje mimético: } \\
\text { Suficiente. } \\
\text { Lenguaje oral: Básico. } \\
\text { Falta de nivelación. } \\
\text { Lenguaje escrito: } \\
\text { Deficiente. }\end{array}$ & $\begin{array}{l}\text { La docente llega al aula después de descanso y espera a los estudiantes en la puerta, saludándolos e indicándoles } \\
\text { que se vayan ubicando en sus puestos. Terminado el recibimiento de los estudiantes, la docente les recuerda el } \\
\text { compromiso que tenían para la clase, el cual consistía en la evaluación del tema trabajado en la clase anterior. } \\
\text { Algunos estudiantes responden asertivamente frente a la evaluación programada, mientras otros le dicen a la docente } \\
\text { a viva voz que no..., a lo que ella les responde ...que era un compromiso de ellos estudiar para la evaluación y que } \\
\text { además el tema lo habían trabajado de varias formas de modo que quedara claro. Les pide que se ubiquen de manera } \\
\text { que queden bien distribuidos en el salón para evitar que aquellos que les gusta fisgonear a los demás, no puedan } \\
\text { hacerlo, porque se vería obligada a anular las evaluaciones de quienes estén implicados. }\end{array}$ \\
\hline $\begin{array}{l}\text { Evaluación no } \\
\text { adaptada a niños } \\
\text { con DC, quienes se } \\
\text { observan apáticos, } \\
\text { aislados. }\end{array}$ & $\begin{array}{l}\text { Una vez organizados les entrega la evaluación en forma individual, recordando las reglas establecidas para presentar } \\
\text { este tipo de pruebas, insistiéndoles en marcar la hoja con el nombre completo y escuchar con atención las instrucciones } \\
\text { para resolver la evaluación. Una vez entregadas todas las pruebas, se ubica frente de los pupitres visualizando los } \\
\text { estudiantes y en las ocasiones en que fue necesario llamó la atención a quienes hablaban o miraban al compañero. } \\
\text { Se observa que las evaluaciones son iguales para todos los estudiantes, no se hace adaptación para los niños con } \\
\text { DC. Durante la prueba los niños con DC se observan ensimismados y desorientados por lo que la profesora se } \\
\text { acerca a ellos y les da instrucciones particulares frente a la cual los niños inicia marcando la hoja con el nombre. } \\
\text { Posteriormente, uno de ellos intenta realizar la prueba mientras que el otro solo escribe el nombre y se pone a producir } \\
\text { sonidos extraños que distraen a sus pares. La profesora le dirige la mirada y el niño mecánicamente hace silencio. } \\
\text { Durante la prueba los niños en general no guardan silencio ni siguen instrucciones al pie de la letra. }\end{array}$ \\
\hline $\begin{array}{l}\text { Diferencias individuales } \\
\text { entre niños con DC. }\end{array}$ & $\begin{array}{l}\text { Terminado el tiempo estipulado, recoge las evaluaciones y rápidamente empieza a leer pregunta por pregunta, } \\
\text { haciendo la corrección de la misma con todos los estudiantes, escuchando las respuestas dadas por ellos, dando la } \\
\text { aprobación o no de las respuestas, corrigiendo donde era necesario. Los niños con DC mantienen una postura de } \\
\text { indiferencia, es decir, no responden como los demás. }\end{array}$ \\
\hline $\begin{array}{l}\text { Interacción con la } \\
\text { docente. }\end{array}$ & $\begin{array}{l}\text { Para finalizar, la docente copia en el tablero un compromiso para la próxima clase que consiste en practicar dictado } \\
\text { de } 15 \text { palabras que contengan la combinación bl y pide a los niños que lo transcriban al cuaderno. Todos lo hacen, } \\
\text { uno de los niños con DC saca solo su cuaderno y comienza a escribir, el otro niño con DC no es capaz de buscar } \\
\text { su cuaderno por sí solo, por tanto, la maestra viene hasta su puesto y le busca el cuaderno indicándole donde debe } \\
\text { hacer la transcripción. De esta manera el niño inicia la actividad, pero termina mucho después que el resto del grupo. }\end{array}$ \\
\hline
\end{tabular}

La última sesión de observación de clases se realiza dos semanas después. En esta sesión resurgió un lenguaje mimético claro y contundente para expresar rabia o descontento por parte de un niño en situación de DC. De allí, no hubo mayor espacio y oportunidad para incentivar el lenguaje oral ni el lenguaje escrito. En esta sesión de clases, los incidentes comportamentales generaron distracción y ruido en el correr de la clase.
Por todo lo observado, se confirma la atención a la diversidad de necesidades en cuanto a las competencias comunicativas; pero, se descuida notoriamente lo planteado en torno a las necesidades de socialización como situación problémica del presente estudio. Tal como se afirma en trabajos previos a esta investigación, muchos docentes no adaptan las estrategias de enseñanza a los niños y niñas con diversidad cognitiva en su proceso de aprendizaje, por lo que los terminan relegando. En tal sentido, se confirman por lo observado ciertos mitos entre 
los cuales destacan que los maestros poseen una errónea concepción respecto a brindar una atención personalizada a los niños y las niñas en desventaja o diversidad cognitiva. De hecho, la falta de motivación de los niños con DC reaparece más como una consecuencia de las malas prácticas docentes que una causa o disposición interna de estos niños. Es así como se confirma lo dicho en Cabero, Córdoba y Fernández (2008), en cuanto a que los cuestionables señalamientos contra la población con necesidades educativas exclusivas son esencialmente por culpa de malos maestros, así como de padres y representantes invisibilizados ante el problema por la falta información y orientación, lo cual se subsanaría, en alguna medida, con la constitución y funcionamiento como comunidad escolar inclusiva y/o comunidad de aprendizaje.

\begin{tabular}{|c|c|}
\hline Unidades de Análisis & Observación \\
\hline $\begin{array}{l}\text { Lenguaje mimético: Pataleta. } \\
\text { Participación activa del niño con } \\
\text { DC a pesar de reacción emocional } \\
\text { y violenta. }\end{array}$ & $\begin{array}{l}\text { La docente inicia haciendo la oración y continúa con un canto de alabanza, a lo que responden los } \\
\text { estudiantes a viva voz. Llama la atención por el desorden en la clase, lo cual repite en muchas ocasiones. } \\
\text { Se observa que uno de los niños con DC participa activamente en la oración y el canto de alabanza, pero } \\
\text { llama la atención que cuando los estudiantes cantan a gritos, él se coloca las manos en las orejas como } \\
\text { si le molestara el exceso de ruido. Terminado el momento de oración, el niño se dirige hacia la parte de } \\
\text { atrás del salón donde se acurruca en el piso y empieza a mover su cabeza de un lado a otro y a hablar } \\
\text { solo. Algunos de sus compañeros le miran y sonríen, otros parecen ya estar acostumbrados a estos } \\
\text { comportamientos y lo ignoran. }\end{array}$ \\
\hline $\begin{array}{l}\text { Lenguaje oral: Débil. Control del } \\
\text { grupo. }\end{array}$ & $\begin{array}{l}\text { La profesora les dice: déjenlo tranquilo, hagan cuenta que no está y sigue con la motivación para el inicio } \\
\text { del tema, la cual hace a través de la pregunta: ¿Qué cuentos conoces o te han narrado? Ante lo cual todos } \\
\text { empiezan a responder a gritos. Algunos se levantan del puesto para ir hasta donde está la profesora con } \\
\text { el fin de hacerse escuchar por ella. La profesora levanta la voz y cuenta hasta tres para que todos vuelvan } \\
\text { al puesto, y les recuerda que deben alzar la mano para pedir el uso de la palabra. Una vez termina de } \\
\text { escuchar algunas respuestas pide a un estudiante que cuente a todos en la clase lo que hizo el domingo } \\
\text { anterior en su casa. El niño tímidamente narra que el domingo fue al río con su familia y que comieron } \\
\text { sancocho y jugaron con una pelota y que en la tarde regresaron a la casa y se durmió temprano porque } \\
\text { llegó muy cansado. La profesora le dice: muy bien y luego explica al grupo el concepto de narración. }\end{array}$ \\
\hline $\begin{array}{l}\text { Incidente de agresión física } \\
\text { del niño con DC a otro niño. } \\
\text { Exhortación docente a la disciplina } \\
\text { escolar y acatamiento del grupo en } \\
\text { general. }\end{array}$ & $\begin{array}{l}\text { En ese momento la clase se ve interrumpida por el grito de una estudiante que dice que el niño con DC le } \\
\text { ha tirado por una oreja. La profesora va hasta su puesto y la revisa y le dice tranquila y la soba, luego le } \\
\text { dice: Usted sabe que a él debemos tenerle paciencia; seguidamente, se dirige al niño con DC y le llama la } \\
\text { atención diciendo: No me les pegue a los niños, porque si no, no la llevo a la misa. Ante este llamado de } \\
\text { atención, la niña con DC se limita a repetir varias veces: no les pegue a los niños, le voy a decir a Alfonso. }\end{array}$ \\
\hline $\begin{array}{l}\text { Lenguaje escrito: Deficiente. } \\
\text { Reiniciada actitud violenta y } \\
\text { desacato a la disciplina por parte } \\
\text { del niño con DC. }\end{array}$ & $\begin{array}{l}\text { Luego del incidente la profesora pide a los estudiantes que abran su libro de texto en la página } 26 \text { y les } \\
\text { pide que transcriban el contenido de la misma. Se observa un poco de desorden en la clase. Algunos } \\
\text { hablan con sus compañeros, otros, empiezan a trabajar en orden. En ese momento la profesora se dirige } \\
\text { a la parte de atrás del salón donde ha permanecido todo el tiempo el niño con DC y le pregunta: ¿Qué } \\
\text { quieres hacer? A lo cual el niño responde: el niño quiere el collar, entonces la profesora la lleva hasta un } \\
\text { pupitre ubicado al lado de su escritorio y lo sienta mientras le entrega un cordón y unas cuentas de colores } \\
\text { y el niño empieza a enhebrar las cuentas. Las enhebra todas y luego las saca y vuelve a empezar. De } \\
\text { pronto, empieza a tirarle las cuentas a los niños; ellos le ponen la queja a la profesora. Esta le llama la } \\
\text { atención y repite las palabras que la profesora le acaba de decir, luego se levanta del puesto para dirigirse } \\
\text { otra vez a la parte de atrás del salón. La profesora la deja empieza a pasar por los puestos para revisar } \\
\text { los avances en el trabajo asignado. En ese momento la clase se interrumpe. }\end{array}$ \\
\hline
\end{tabular}




\section{PROCESAMIENTO DE LA ENTREVISTAS A EXPERTOS}

A partir de lo observado en el salón de clases, contribuyó a definir ciertos temas a tratar durante la entrevista a dos expertos en Psicopedagogía y Educación Especial. Así se comenzó preguntándoles: ¿Qué papel juega una comunidad de aprendizaje dentro de los procesos de enseñanza y aprendizaje de la lectoescritura, especialmente con niños en situación de discapacidad cognitiva?

En efecto, puede observarse como unidades de análisis emergentes de lo dicho por el Experto A entrevistado, quien considera las CoA como necesarias para que todas las personas en torno al desarrollo del niño y su aprendizaje tomen su rol y desde cada perspectiva hagan un aporte: la familia, el docente, los compañeros, la comunidad en general. Más específicamente, el Experto A sostiene que las comunidades de aprendizaje juegan un papel importante ya que hay muchos tabús frente a las y los niños con necesidades especiales y el cómo atenderlos; cómo llegar a ellos y cómo desarrollar en ellos estas capacidades (en lectoescritura).

El Experto B destacó en su respuesta que las CoA sirven para superar una tradición en la cual, básicamente los padres entregaban esa responsabilidad a la escuela... (cuando) hoy en todo sentido evolutivo se entiende $y$ asi lo marca entre otras las mismas normas (legales) y colombianas que la responsabilidad educativa es del Estado, la Sociedad y la Familia. Por tanto, puntualiza el Experto B, en esa misma condición de triada significa que los mismos fines los logramos en la medida en que interaccionemos, organizados como comunidad. Este mismo autor, asegura que las comunidades de aprendizaje sirven para, un ejercicio más disciplinar e interdisciplinar, sectorial e intersectorial. Púes, no sólo se abre la posibilidad o implica un ejercicio (del oficio de enseñar) directamente al estar con ellos (los niños especiales). Así mismo, se resalta que otros tienen la obligación de contribuir de alguna manera de acuerdo a los contextos en los campos de la formación, en los saberes que se recopilan de la investigación (sobre) los tratamientos que pueden existir en el mismo desarrollo de las aulas escolares.

Pasando a la segunda pregunta, se les increpó a los entrevistados sobre: ¿Qué experiencias significativas ha conocido usted respecto a la comunidad de aprendizaje como apoyo directo a los procesos del aula común en alguna institución? El Experto A destacó su participación directa en la organización de un grupo se empezó (por) capacitar primero a las maestras (...) seguidamente, se reunieron a los padres de familia de estos niños (que presentaban algún tipo de discapacidad o alguna necesidad educativa especial. Luego de sensibilizarlos respecto a las posibilidades de mejora en el desarrollo de esta población especial, se empezó a hacer ese proceso muy hermoso (cuando) se empezaron a vincular a los papás en las actividades pedagógicas... y la experiencia maravillosa de los compañeros y el rol que juegan los otros compañeros, los estudiantes en ese desarrollo de esos niños.

Así, relata el Experto A, como poco a poco se fue formando esa comunidad y se fue alimentando y fue creciendo. Tal vez, lo más satisfactorio del éxito de tal experiencia fue destacar como hubo: Varios casos de niños que ingresaron en sala cuna con diagnóstico desesperantes, donde los niños no iban a caminar, que no iban a poder hablar, que les daban muy poco tiempo de lucidez y la interacción, las relaciones sociales, el amor, la aceptación que se empezó a gestar en esta comunidad de aprendizaje fue creciendo y fue creciendo que actualmente esos niños pasaron, tramitaron a educación formal con bases académicas, con capacidades. 
Continuando con el aporte del Experto B, las experiencias en la promoción de las comunidades de aprendizaje abarcan todos los estudiantes en algunos programas, por ejemplo; para el mejoramiento de las competencias en matemáticas y lenguaje. Más particularmente puntualiza que, se genera abajo y arriba es decir (desde) el entorno en que está la escuela, se construyeron esas comunidades con todos los directivos y todos los docentes que están en ese entorno. En cuanto a las CoA para la población que está en discapacidad, se destaca que el Experto $\mathrm{B}$ cree que es un proceso que en el país (Colombia) está en este momento evolucionando, por lo menos se está orientando a que estas se atiendan. Pues, aquí se tenían... establecimientos especializados para el tema. Hoy que se rompe ese esquema y lo que se busca es tratar aquí para este caso equipos interdisciplinarios.

Pasando a la tercera pregunta, se inquirió: ¿qué relevancia tiene el nivel educativo de los miembros de una comunidad de aprendizaje? En el caso específico de incorporar a los padres de familia de estos niños en situación de discapacidad cognitiva: ¿Cree usted que necesitan cierto nivel académico para poder participar de una comunidad? o ¿Cualquiera puede hacerlo?

El Experto A no duda en señalar, al respecto, que, cualquier persona puede participar en estas comunidades de aprendizaje. Lo importante es identificar actores y también los roles, dice. Antes que un perfil académico, en su opinión, lo importante es que tengan el deseo de aprender. Así mismo, destaca que es fundamental que deseen trabajar, que deseen multiplicar, ser esos agentes multiplicadores para el logro de estos objetivos. El Experto $\mathrm{B}$ es aún más radical cuando responde: yo creo que ese es el paradigma a vencer, que se cree que si no hay expertos no se puede hacer cualquier trabajo de comunidad. Pero aquí independientemente, los padres de familia simplemente en su buena orientación constituyen un soporte fundamental. Por otra parte, emerge el aporte como del Experto B en cuanto a que, (la) formación universitaria ha sido muy individual, y eso hace incluso que los mismos profesionales e investigadores y demás no (se) reúnan y compartan realmente todas esas resultantes de estudios. Pero, independientemente, los padres de familia son quienes comparten. En tanto son los que conocen, los que ven, los que hacen, los que observan, los que aprecian. Continúa afirmando, pero, se requiere organización y administración. Ninguna comunidad funcionaria si no tiene una estructura, un orden, una manera de hacerlo y esa es la apuesta que se tiene que hacer hoy el sector educativo.

Continuando con la cuarta pregunta, ¿Qué tan factible puede resultar la implementación de una comunidad de aprendizaje como estrategia curricular innovadora para la enseñanza aprendizaje de niños y niñas con discapacidad cognitiva? Según el Experto A, claro que es super, super interesante y sería estupendo donde se lograra implementar como una estrategia curricular para el aprendizaje.

Para el Experto B el problema no es lo factible sino lo necesario, pues, roles no podemos hacerlo. Eso implica que las mismas instituciones educativas tienen que abrir sus puertas y naturalmente tienen que convocar muchos más actores. En efecto, más para aquellas personas excluidas en el tiempo y que requieren unas estrategias pedagógicas mucho más pertinentes, más propias e incorporarlas $y$ reconocerlas como personas eso hace con mayor razón la necesidad de tener una comunidad de aprendizaje.

Más concretamente, señala el Experto $B$, no basta con recibir los niños especiales en las aulas de clase sino, con qué nivel de atención los vamos a tratar, y que oferta le tenemos también, 
para que ellos aun estando en un salón de clase no se sientan excluidos de la misma manera. $\mathrm{Y}$ los maestros que tienen el mayor protagonismo en la promoción de las comunidades de aprendizaje que tienen su acción directa, con mayor razón no se deben invisibilizar ante el problema.

\section{PROCESAMIENTO DE LA ENTREVISTA A LA DOCENTE}

Se entrevisto a la docente titular del grado Primero en la institución educativa objeto de estudio. Se iniciar con la pregunta ¿Cree usted que se justifica adelantar los esfuerzos tendientes a crear una comunidad de aprendizaje para auxiliar el proceso formativo de niños con diversidad cognitiva? (PAUSA) Particularmente, ¿Cómo podrían atacarse los problemas de estos niños en cuanto a las competencias comunicativas y sociales? La docente muy seria y puntualmente respondió: claro, sería maravilloso que ese tipo de innovación llegara al Colegio porque...permite la interacción de los padres de familia con los docentes para apoyar los procesos pedagógicos... Además, puntualizó que, facilitaría a los docentes de aula manejar otro tipo de herramientas, conocer estrategias que han implementado algunos padres $u$ otros docentes y... sirve como apoyo para el proceso que se hace con estos niños en el aula.

Con la siguiente pregunta, se le increpó: ¿Cómo cree que podrían atacarse los problemas de estos niños en situación de diversidad cognitiva respecto a sus competencias comunicativas y sociales? La docente respondió de forma espontánea y serena que, para eso hace falta el apoyo de un equipo interdisciplinario para las instituciones. Además, se requieren los implementos y el material humano capacitado para desarrollar estos procesos con el niño. También, sugiere que aún los niños en situación DC difieren en sus habilidades por lo que a cada quien, se le debe explorar y mirar a ver en donde están para poder fortalecer. Como aclaró antes: aquí no se sabe a ciencia cierta qué tipo de discapacidad presentan los niños. Algunos se saben qué es, apenas, por lo que los padres dicen y otros que tampoco traen los soportes médicos.

Respecto a la siguiente pregunta, ¿Cree usted que las comunidades de aprendizaje serían un instrumento eficaz o un apoyo real para su trabajo en el aula común? La docente en su franco concepto, cree que sí. Una posición que fundamenta al empezar a abrir nuevos espacios para poder encontrar herramientas que conlleven a facilitar los procesos con los niños con atención inclusive. Ante lo cual aclara que, se habla de inclusión... porque se le abren las puertas de las instituciones, pero realmente no es inclusión.

Continua con la entrevista, se le pregunta: De acuerdo a su experiencia como docente de aula común, ¿cuáles competencias comunicativas desarrollan con mayor facilidad los niños en situación de diversidad cognitiva $(D C)$ ? La docente resalta la oralidad como las más importante, aclarando posteriormente su respuesta: pero que la competencia escrita realmente no, los niños es poco lo que escriben... y las competencias lectoras tampoco la desarrollan.

Luego se le pregunta, ¿cómo describiría usted las condiciones curriculares de la institución educativa para impartir una enseñanza efectiva a niños y niñas en situación de diversidad cognitiva? Denotando seguridad y conocimiento de la realidad de la institución, la docente increpa, empezando hay que decir que el colegio no cuenta con un plan de estudio o una masa curricular que contenga adaptaciones para este tipo de población, partimos de cero. En cuanto a las calificaciones, como no pueden avanzar por su condición cognitiva y tampoco pueden reiniciar el año, entonces se les asigna 
como calificación lo básico que es tres. En cuanto al acompañamiento a los niños con DC, el profesor se para ahí... pero no se le dedica el tiempo que realmente se le debe dedicar. Tampoco (continua la docente), se particulariza en la enseñanza aprendizaje.

Luego se le pregunta, ¿cabría algún cambio en su opinión, particularmente, en cuanto a las condiciones administrativas del currículo o del personal directivo de la institución? Especifique. Con una seria preocupación e incertidumbre de la docente, por lo que llama el trasfondo social, riposta: La inclusión como dice el papel no es así, es decir, es que se están recibiendo y sí se están atendiendo, pero el trasfondo es otro. Pues, ella ha aclarado antes que, para ser incluyente se tiene que prestar un servicio de calidad y verdaderamente atender las necesidades de esa población.

Luego se le pregunta, ¿cómo aprecia u observa usted la actuación general o el desempeño de estos niños con DC en el aula común? A lo que la docente responde: Pues en el aula ellos no se ven apáticos, no se ven alejados ni se aíslan, tienen una interacción con el grupo. En la parte social buena, los niños son respetados y aceptados por sus compañeros. Lo deficiente de la actuación general del niño con DC, la misma docente lo expresa así: la dificultad radica en el proceso a la hora de abordar las áreas de conocimiento, porque los niños se sienten como frustrados. En términos más descriptivos agrega: porque ellos ven que los otros hacen y ellos se quedan mirando la hojita. Se distraen porque no encuentran qué hacer y nada les llama la atención.

Tal vez, el mayor problema para la actuación general del niño con DC gira en torno a un aula común sobresaturada de estudiantes. Pues, como también señala la docente, un docente tiene que atender a 30 estudiantes en un aula de clase, a qué horas saca tiempo para dedicarle a estos niños que requieren de más acompañamiento de la profesora.

Continuando se le pregunta, ¿cómo espera en su experiencia que sea la reacción u opinión de los padres y representantes de niños, en situación de diversidad cognitiva en cuanto a la inclusión en un aula común o integrada junto con los demás niños? La docente responde refiriendo que los padres de familia no se han apropiado de ese concepto (inclusión), simplemente siguen dejando los niños para que sean cuidados. Peor aún, puntualiza con un tono de decepción que, así pasa paradójicamente no sólo con ellos sino con todos. Esta actitud de indiferencia también se pone en acción, pues, a la hora de hacer presencia, cuando se requiere al Padre de Familia para adelantar algún proceso en lo pedagógico y en lo comportamental, rara vez asisten. Finaliza la docente añadiendo que, falta mucho compromiso, realmente a los padres más que compromisos les falta formación.

Luego se le pregunta, ien su experiencia docente, ¿Qué se demanda para llevar a cabo lo que hoy día tiende a llamarse Comunidades de Aprendizaje o Comunidades de Práctica dentro de la institución educativa? (PAUSA) ¿Cuáles materias, objetivos y actividades deberían ser enfocadas y prioritarias, dentro de estas comunidades?

En forma entusiasta, la docente responde: Primero, yo creo que las comunidades de aprendizaje deben estar orientadas a la formación de cómo guiar a un Padre de familia; de cómo darle una orientación pedagógica que sea real, que sea efectiva. En cuanto a las materias prioritarias, la misma docente destaca que: a través de estas comunidades de aprendizaje... la Lengua Castellana es fundamental porque... a través de sus competencias puede llegar hasta desarrollar unas competencias matemáticas, de las ciencias naturales, las sociales. Porque la 
parte artística va como así en el engranaje con las otras.

\section{DISCUSIÓN Y CONCLUSIÓN}

Nótese que la preocupación común de la docente a todo lo largo de la entrevista es resaltar como las CoA contribuirían a rescatar la interacción entre PyR tanto como entre estos y el docente. Así, se coincide con Lozada y Moreno (2006) tanto como con García Delgado (2005) que, desde el punto de vista social y antropológico, se reconocen o reafirman los principios básicos: (a) El principio de relacionabilidad; (b) el principio de alteridad; y (c) el principio de dialogicidad, que se explican por sí mismos. Además, se logra el hito en el cual se encuentra la humanidad que demanda de muchas voluntades en educación.

Más aún, queda reafirmada la máxima de Poole (2003) de que, los profesores necesitan a los padres. Máxime aun cuando nuevamente acierta el reseñar: "una de las grandes debilidades de la educación, en la (actual) época al menos, es el distanciamiento que existe entre el hogar del alumno y la escuela donde este estudia." (p.159). En efecto, hoy día se impone, como pautan Díaz-Barriga Arceo y Hernández Rojas (2010), la idea del trabajo cooperativo como lo exigen las comunidades de aprendizaje.

A modo de triangulación de fuentes y datos, es evidente la preocupación común entre los expertos y los docentes entrevistados sobre varios aspectos centrales; los cuales se erguen como hallazgos del presente estudio. Una de las cuales queda reafirmada como una de las grandes debilidades de la educación que persiste actualmente y que denunciara Poole (2003), específicamente, los entrevistados coinciden en cuestionar: "el distanciamiento que existe entre el hogar del alumno y la escuela donde este estudia hasta el punto que se ha llegado a producir una auténtica separación entre lo que sucede dentro de la casa y lo que pasa en la escuela" (Poole, 2003, p.59)

Como agrega Harch (citado en Poole, 2003), al denunciar la permanente separación entre el hogar y la escuela, se ha perdido la oportunidad para "tratar con aquellos estudiantes que están en riesgo, para reducir posibles deserciones, para mejorar los resultados escolares y (significativamente) las relaciones entre la escuela y la comunidad en la que se engloba" (p.60). Así, los entrevistados reconocen esta realidad tanto como la necesidad de incorporar a los padres más que pensar en la factibilidad de las comunidades en torno a los niños en situación de discapacidad cognitiva del plantel seleccionado, reafirmando la importancia del triángulo conformado por los padres, el docente y los estudiantes; el cual, de cultivarse, robustecería de forma significativa las experiencias que viven los estudiantes en la institución educativa.

En efecto, los expertos y la docente entrevistados también coinciden en fortalecer las comunidades de aprendizaje como estrategia de apoyo; así la definen Díaz-Barriga Arceo y Hernández Rojas (2010) al también denominarlas comunidades de práctica y "estrategias de administración de recursos que también llegan a ubicarse en el plano motivacional- afectivo" (p.377). También reafirman los entrevistados la idea esencial de la comunidad de aprendizaje, en principio, como un grupo de personas que interaccionan socialmente porque comparten temporal o permanentemente una serie de intereses comunes" (García Aretio, 2003, p.171).

Al respecto, coincidieron con Foster (citado en García Aretio, 2003) en cuanto al "elemento comunicacional, ... esencial dentro del concepto de comunidad" que permiten ofacilitan las nuevas tecnologías de la información y la comunicación; de allí, reafirman los entrevistados, la posibilidad de operar en ambientes virtuales como sugiere 
Fainholc (2009). Igualmente, extraña que no emergió cuestionamiento al ciberespacio para las comunidades de aprendizaje.

Igualmente, los expertos y la docente han compartido experiencias vivenciales que contrastan cierto postulado teórico o generalización sobre las CoA. Según Shopino y Levine (citados en García Aretio, 2003), las CoA deberían mantenerse apegadas a algunas características básicas como: "(a) El estar organizados profesores y alumnos en grupos pequeños" (p.173). Nótese, entonces, que según tal fuente referencial no entran como miembros los padres. Una cuestión incompatible con la opinión de los entrevistados ni con la de otros autores. La preocupación en la que sí coinciden los entrevistados con la fuente en referencia es que toda CoA: debe mantenerse apegada a otras características básicas de:

(b) Promover la integración curricular; (c) Ayudar a los estudiantes a mantener redes de apoyo; (d) Facilitar escenarios para la socialización de los estudiantes; (e) Facilitar la colaboración y unión de los propios profesores entre ellos de manera visible; (f) Implica a profesores y estudiantes en los resultados del aprendizaje de estos; y (g) Promover ambientes para la crítica (García Aretio, 2003, p.173).

Así mismo, se identificaron coincidencias entre las respuestas de los entrevistados con la postura de García Aretio (2003) de que no hace falta un nivel académico para pertenecer a una comunidad de aprendizaje. Más bien, destacan la conveniencia de diferentes experiencias personales valoradas por sus contribuciones y apoyo a los demás, "un objetivo compartido respecto al avance del grupo en conocimiento y habilidades; un énfasis en como aprender" (p.182).
Finalmente, queda confirmada por la opinión de los entrevistados y los autores consultados en esta investigación que la noción de diversidad antes que, de discapacidad cognitiva, independientemente de que exista o no retraso mental (Cabero, Córdoba y Fernández, 2008). Así, se supera la noción de la diversidad cognitiva como condición biológica inalterable para entenderse más como resultado de la interacción del niño o la niña afectada con un medio o entorno psicosocial y educativo que poco o mucho le puede favorecer.

\section{REFERENCIAS BIBLIOGRÁFICAS}

Cabero, J. Córdoba, M. y Fernández, J.M. (2008). Las TIC como elementos en la atención a la diversidad. En J. cabero, M. Córdoba y J. M Fernández (Coords) Las TIC para la igualdad. Nuevas Tecnologías y atención a la diversidad (pp. 15-35). Sevilla, España: Eduforma-Magisterio.

Congreso de Colombia. Decreto 2082 (1996). Reglamento de atención educativa para personas con limitaciones o con capacidades o talentos excepcionales. Bogotá, Colombia: El Congreso.

Congreso de Colombia. (1997). Ley 361 de integración social de la persona con discapacidad. Bogotá, Colombia: EI Congreso.

Consejería Presidencial para la Política Social. (2002). Plan nacional de atención a las personas con discapacidad. Manual operativo. Bogotá, Colombia: Presidencia de la República.

Díaz-Barriga Arceo, E. y Hernández Rojas, G. (2010). Estrategias docentes para un aprendizaje significativo. Una interpretación constructivista. $\quad\left(3^{a} \quad\right.$ edición.) México: McGrawHill. 
Fainholc, B. (2009). El fortalecimiento de las competencias comunicativas $y$ la trascendencia pedagógica de las tecnologías de la enseñanza. En A. Fernández y A.B. Fundación Unicornio. (2013). Discapacidad mental. (Documento en línea). Disponible: http//www. ladiscapacidad.com/discapacidadcognitiva/ discapacidadcognitiva.php.

Gamboa-Suárez, A. A. (2016). Docencia, investigación y gestión: Reflexiones sobre su papel en la calidad de la educación superior. Revista Perspectivas, 1(1), 81-90. https://doi.org/10.22463/25909215.973.

García Aretio, L. (2003). Comunidades de aprendizaje en entornos virtuales. La comunidad iberoamericana de la CUED. En B. Álvarez (ed.) y M. Barajas (Coord.) La tecnología educativa en la enseñanza superior. Entornos virtuales de aprendizaje. (pp. 171-202)

García Delgado, G. (2005). El mundo del maestro y la sociedad del aprendizaje permanente. Venezuela: UNESR.

García-Quintero, C., \& Villamizar Suárez, G. (2017). Análisis fenomenológico de la conciencia del docente a partir de sus prácticas evaluativas. Revista Perspectivas, 2(2), 49-59. https://doi. org/10.22463/25909215.1313

Hernández Sampieri, R. Fernández Collado, C. y Baptista Lucio, P. (2010). Metodología de la investigación. (5a . Ed.) México: McGrawHill.

Landeau, R. (2007). Elaboración de trabajos de investigación. (J. Sambrano, rev.tec.). Caracas. Alfa.
López-García, J. D., \& Gutiérrez-Niño, D. (2018). Efecto del uso de la herramienta "realidad aumentada" en el rendimiento académico de estudiantes de Educación Básica. Revista Perspectivas, 3(1), 6-12. https://doi. org/10.22463/25909215.1464.

Lozada, A. y Moreno, H. (2006). Competencias básicas por áreas aplicadas al aula. ( $3^{\mathrm{a}}$. Ed.) Bogotá, Colombia: SEM-ABC del Educador.

Méndez- Omaña, J. P., \& Jaimes- Contreras, L. A. (2018). Clima social familiar e impacto en el rendimiento académico de los estudiantes. Revista Perspectivas, 3(1), 24-43. https:// doi.org/10.22463/25909215.1422.

Ministerio de Educación Nacional (2006). Orientaciones pedagógicas para la atención educativa a estudiantes con discapacidad cognitiva. (Documento en línea). Disponible: http://www.colombiaaprende.edu.co/ftml/ micrositios/17icles-320691-archivo_5pdf.

Poole, B. J. (2003). Tecnología educativa. En B. Martínez de Murgía (trad.) y M.A. AguarelesArrovo (rev.tec.). Como desarrollar una práctica docente competitiva. (2 ${ }^{\mathrm{a}}$. ed.) Bogotá, Colombia: McGrawHall-Colección Docente del Siglo XXI.

Stainback, S., Stainback, W. y Jackson, H.J. (1999). Hacia las aulas inclusivas. En S. Stinback y W. Stainback (Comps.). Aulas inclusivas, (pp. 21-35). Madrid: Narcea. 